\title{
Adaptive Estimation of Measurement Bias in Three-Dimensional Field Sensors with Angular-Rate Sensors: Theory and Comparative Experimental Evaluation
}

\author{
Giancarlo Troni and Louis L. Whitcomb \\ Department of Mechanical Engineering \\ Johns Hopkins University \\ Baltimore, Maryland 21218, USA \\ Email: giancarlo.troniegmail.com, llwejhu.edu
}

\begin{abstract}
Three-axis magnetometers and three-axis accelerometers are widely used sensors for attitude estimation, yet their accuracy is limited by sensor measurement bias. This paper reports a novel methodology for estimating the sensor bias of three-axis field sensors (e.g. magnetometers and accelerometers). Our approach employs three-axis angular velocity measurements from an angular-rate gyroscope to estimate the three-axis field sensor measurement bias that, when properly calibrated, can significantly improve attitude estimation. We report three methods implementing this approach based on batch linear least squares, real time Kalman filter, and real time adaptive identification. Our methods impose less restrictive conditions for the movements of the instrument required for calibration than previously reported methods, do not require knowledge of the direction of the field (e.g. the local magnetic field) or the attitude of the instrument, and also ensure convergence for the estimated parameters. The proposed methods are evaluated and compared with the previously reported methods with numerical simulation and in comparative experimental evaluation with sensors onboard an underwater robot vehicle.
\end{abstract}

\section{INTRODUCTION}

Magnetometers, accelerometers, and angular-rate gyroscopes are widely used in many applications including vehicle navigation systems (e.g., space, air, ground, and marine vehicles) and in personal electronics devices such as smart-phones and tablet devices. Magnetometers are commonly used to measure Earth's local magnetic field vector and thus determine the device heading. Accelerometers are commonly used to measure the device's inclination with respect to the Earth's local gravity vector and thus determine the local horizontal plane and vertical direction - i.e. roll and pitch. Angular-rate gyroscopes measure the angular rotation rate of the device. Currently many integrated devices incorporate angular-rate gyroscopes combined with accelerometers and magnetometers to enable estimation of the full attitude (e.g., heading, pitch and roll) of the instrument. Several methods are available to estimate the attitude with these sensors - see the recent Crassidis et al.'s review of attitude estimation methods [6]. All of these sensors are affected by biases, scale factors, and non-orthogonality of their measurements. Sensor calibration is critical for accurate performance of attitude estimation with these devices.

Magnetometers are highly affected by magnetic field disturbances that can cause erroneous measurements that can change over time. For magnetometers applications in which continuous calibration is required for accurate performance, real time, adaptive sensor bias estimation is desirable.

Several approaches for magnetometers calibration have been reported that estimate the calibration parameters without the use of additional references sensors. Traditional approaches include approximated solutions such as the conventional heading swing method, e.g., [5]. Recently, several reported methods have addressed more rigorously the three-axis magnetometer calibration problem. The problem of self-calibrating a three-axis magnetometer without external reference, can be formulated as a minimization problem that requires iterative methods to solve for the calibration parameters. For estimating magnetometers bias, Gambhir proposed a "centered" approximation that can be solved with linear least squares [8]. Alonso and Shuster proposed, the "TWOSTEP" method, that uses Gambhir's solution as initialization to an iterative second step for estimating the sensor bias [2], and, as reported in a later work, also estimates the scale and orthogonality factors [3]. In a similar approach, Vasconselos et al. [20] formulate the problem as an ellipsoid fitting problem, and solve it using an iterative maximum likelihood estimate (MLE) method. For practical implementation in different applications there are some limitations to these methods. In general, all of these calibration methods require large angular movements of the instrument to measure a large section of the magnetic sensor output for an accurate calibration. For better performance it is necessary to know accurately the local magnitude of the Earth's magnetic field. This value can be calculated by magnetic field models, e.g. [16], but can present large error in environments with unmodeled magnetic distortions (e.g., due to buildings or other local magnetic anomalies). Finally these methods are formulated for batch estimation and are not practical for continuous real time operation. 
Many applications require the ability to perform the field sensor calibration in continuously real time due to (1) the calibration parameters changing during operations (e.g., due to change in vehicle payload) or (2) local field disturbances that render it impossible to estimate the calibration parameters before operation (e.g., for an underwater vehicle deployed from a large steel ship). Crassidis and Lai, [7], propose an extension to the TWOSTEP method based on the extended Kalman filter (EKF) and the unscented Kalman filter (UKF) to estimate in real time the sensor bias, scale, and orthogonality factors. Ma and Jiang proposed an alternative method based on the UKF, [15], and Guo at al., [9], reported an EKF approach. These methods exhibit some of the same problems noted for the batch methods, and also do not ensure convergence of the estimated parameters to the true values.

Although inertial sensors are commonly available with magnetometers in a combined instrument package, most previous reported methods for calibrating magnetometers do not utilize these additional sensors. $\mathrm{Li}$ and $\mathrm{Li}$ [14] and Kok et al. [13] make use of the accelerometers to measure the local gravity vector to propose methods based on dot product corrections to estimate the magnetometer calibration. The drawback of these approaches based on acceleration measurements is that translational accelerations of the instrument can perturb the measurements introducing errors in the the magnetometer calibration.

As noted with previous methods there are several barriers that limit their wide implementation: they require large angular movements, they require accurate knowledge of the magnitude of the local magnetic field, and they are only available as batch methods or do not ensure convergence properties. The current paper reports a novel approach to the problem of accurate real time adaptive estimation of the sensor bias in threedimensional field sensors. Our approach employs three-axis angular velocity measurements from an angular-rate gyroscope to estimate the field sensor bias of a three-axis magnetometer or three-axis accelerometer. Three methods are proposed based on this novel approach: one based on linear least squares, one Kalman filter approach, and one novel adaptive identification approach. Our main motivation is for the problem of magnetometers sensor bias, but it can also apply to other field sensors such as three-axis accelerometers. The novel proposed solution does not, at present, estimate scale and orthogonality factors, but can be extended to these cases. But the proposed approach presents several advantages: (1) it is less restricted in the angular movements required, (2) it does not require local field information, such as the magnitude and/or direction of the local magnetic field, (3) it is implementable in real-time, and (4) it has provable convergence properties.

This paper is organized as follows. In Section II we give a brief overview of notation and describe the sensor error model. In Section III we describe our proposed methods for sensor bias estimation. In Section IV we describe our experimental setup and we report a comparative numerical simulation and comparative experimental evaluation of the performance of the different sensor bias estimation methods.
Section VI summarizes and concludes.

\section{BACKGROUND}

\section{A. Mathematical Notation}

We represent the rigid body attitude using the rotation matrix $R(t) \in S O(3)$ describing the orientation of the instrument frame $V$ with respect to an inertial (world) fixed frame $W$.

Let $\omega=\left[\omega_{x}, \omega_{y}, \omega_{z}\right]^{\top} \in \mathbb{R}^{3}$ and define $S: \mathbb{R}^{3} \rightarrow \mathbb{R}^{3 \times 3}$

$$
S(\omega)=\left[\begin{array}{ccc}
0 & -\omega_{z} & \omega_{y} \\
\omega_{z} & 0 & -\omega_{x} \\
-\omega_{y} & \omega_{x} & 0
\end{array}\right] .
$$

The euclidean vector norm is defined as usual as $\|x\|=\left(x^{\top} x\right)^{1 / 2}$, with $x \in \mathbb{R}^{3}$.

\section{B. Sensor Error Model}

Three-axis magnetometers and accelerometers are widely used in navigation applications. Measurements from these sensors are subject to systematic errors due to sensor bias, scale factor and (lack of) orthogonality. We consider the usual model for sensing bias

$$
x(t)=\bar{x}(t)+b
$$

where $\bar{x}(t) \in \mathbb{R}^{3}$ is the true field value in the instrument reference frame, $x(t) \in \mathbb{R}^{3}$ is the measured field value in the instrument reference frame, and $b \in \mathbb{R}^{3}$ is an unknown constant sensor bias.

Multi-axis field sensors such as magnetometers and accelerometers measure, in instrument coordinates, the Earth's local magnetic field or gravity vector, respectively, which are each considered to be locally constant and fixed with respect to the inertial world frame of reference. The true fixed worldframe field vector $x_{0}$ is related to the instrument-frame sensor measurement of the field $\bar{x}(t)$ by

$$
x_{0}=R(t)(x(t)-b) .
$$

In the case that the attitude of the instrument, $R(t)$, and the true field vector, $x_{0}$, are both known accurately, then solving for the unknown sensor bias, $b$, is trivial.

In many practical cases, however, such as the case of the ubiquitous micro-electro-mechanical systems (MEMS) inertial measurement units (IMUs) that are widely used in vehicle navigation systems, $R(t)$ is not directly instrumented - thus the trivial calibration solution is not applicable for these devices. MEMS IMUs are typically equipped with a 3-axis magnetometer, a 3-axis accelerometer, a 3-axis angular-rate gyroscope, and a temperature sensor.

\section{Proposed Methods}

In this section, we report a novel approach to estimate the sensor measurement bias error in 3-axis field sensors (e.g. magnetometers) based on the instrument-frame angularrate measurements (e.g., from angular-rate gyroscopes). Three methods based on this approach are proposed. 


\section{A. System Model}

Differentiating (3), yields

$$
0=\dot{R}(t)(x(t)-b)+R(t) \dot{x}(t) .
$$

Using the the standard equation $\dot{R}(t)=R(t) S(w(t))$ and rearranging the terms, we have

$$
\dot{x}(t)=-\omega(t) \times(x(t)-b),
$$

where $w(t)$ is the measured angular-rate in instrument coordinates, and $\times$ is the standard cross product operator. Note that the instrument attitude, $R(t)$, does not appear in (5).

We wish to estimate the constant unknown sensor bias, $b$, for the system (5) from the signals $\omega(t)$ and $x(t)$. The proposed solutions include (i) a batch least squares method, (ii) a real time Kalman filter method, and (iii) a novel real time adaptive identification method.

\section{B. Linear Least-Squares for Sensor Bias Calibration}

The unknown sensor bias, $b$, can be estimated with linear least squares estimation. The sum of squared residuals cost function is

$$
S S R(b)=\sum_{i=1}^{n} \frac{1}{\sigma_{i}^{2}}\left|\dot{x}_{i}+\omega_{i} \times\left(x_{i}-b\right)\right|^{2},
$$

where $\sigma_{i}$ is variance of the measurement, and each measurement $x_{i}$ is a discrete sample measurement (e.g., $x_{i}$ represents a discrete-time sampling of $x(t)$ ). The linear least squares estimate for $b$ is given by

$$
\begin{aligned}
\hat{b} & =\underset{b \in \mathbb{R}^{3}}{\arg \min } \operatorname{SSR}(b) \\
& =\left(\sum_{i=1}^{n} \frac{1}{\sigma_{i}^{2}} W_{i}^{2}\right)^{-1}\left(\sum_{i=1}^{n} \frac{1}{\sigma_{i}^{2}} W_{i} y_{i}\right),
\end{aligned}
$$

where $W_{i} \in \mathbb{R}^{3 \times 3}$ is the skew-symmetric matrix from the measurements $\omega_{i}, W_{i}=S\left(w_{i}\right)$, and $y_{i} \in \mathbb{R}^{3}$ is the calculated vector from the measurements, $y_{i}=\dot{x}_{i}+\omega_{i} \times x_{i}$. The solution (7) exists when the set of measured angular velocity vectors, $\left[\omega_{1}, \omega_{2}, \cdots \omega_{n}\right]$ are not all collinear, in consequence, $\left(\sum_{i=1}^{n} W_{i}^{2}\right)$ is invertible.

A drawback of this approach is that it requires the signal $\dot{x}(t)$ that is not directly instrumented in magnetometers and accelerometers, and thus this approach requires (possibly noisy) numerical differentiation of the sensor measurement $x(t)$.

\section{Kalman Filter for Sensor Bias Calibration}

The system (5) can be rewritten as

$$
\underbrace{\left[\begin{array}{c}
\dot{x} \\
\dot{b}
\end{array}\right]}_{\dot{\Phi}}=\underbrace{\left[\begin{array}{cc}
-S(\omega) & S(\omega) \\
0 & 0
\end{array}\right]}_{A(t)} \underbrace{\left[\begin{array}{l}
x \\
b
\end{array}\right]}_{\Phi},
$$

with the measurement model

$$
z=\underbrace{\left[\begin{array}{ll}
I & 0
\end{array}\right]}_{H}\left[\begin{array}{l}
x \\
b
\end{array}\right],
$$

we can define the following time-varying system

$$
\begin{aligned}
\dot{\Phi}(t) & =A(t) \Phi(t)+\nu_{1}(t), & & \nu_{1}(t) \sim \mathcal{N}(0, Q), \\
z & =H \Phi+\nu_{2}(t), & & \nu_{2}(t) \sim \mathcal{N}(0, R) .
\end{aligned}
$$

After a discretization of the continuous-time system the sensor bias estimation can be solved with a standard discrete-time Kalman filtering implementation [11], [19] that does not require differentiation.

\section{Adaptive Identification for Sensor Bias Calibration}

The unknown sensor bias, $b$, can be estimated on-line with a novel adaptive identification algorithm which, to the best of our knowledge, has not been previously reported. A possible advantage of this adaptive approach is that it does not require numerical differentiation of the sensor measurement $x(t)$.

Consider the following adaptive observer for the plant of the form (5)

$$
\begin{aligned}
\dot{\hat{x}}(t) & =-\omega(t) \times(\hat{x}(t)-b)-k_{1} \Delta x, & \hat{x}(0) & =\hat{x}_{0} \\
\dot{\hat{b}}(t) & =k_{2}(\omega \times \Delta x) & , \hat{b}(0) & =\hat{b}_{0},
\end{aligned}
$$

where estimation errors are defined as

$$
\Delta x(t)=\hat{x}(t)-x(t), \quad \Delta b(t)=\hat{b}(t)-b .
$$

Given the measured angular-rate signal, $\omega(t)$, and biased 3axis field sensor measurement, $x(t)$, our goal is to construct an estimate of $\hat{b}(t)$ of the unknown sensor bias parameter $b$ such that: 1) all signals remain bounded, and 2) $\hat{b}(t)$ converge asymptotically to $b$, i.e. $\lim _{t \rightarrow \infty} \Delta b(t)=0$.

Before deriving the adaptive identifier, we first review some results required later.

Definition 1 (Persistent Excitation (PE) [18]). A matrix function $\mathcal{W}: \mathbb{R}^{+} \rightarrow \mathbb{R}^{m \times m}$ is persistently exciting (PE) if there exist $T, \alpha_{1}, \alpha_{2}>0$ such that for all $t \geq 0$ :

$$
\alpha_{1} I_{m} \geq \int_{t}^{t+T} \mathcal{W}(\tau) \mathcal{W}^{\top}(\tau) \mathrm{d} \tau \geq \alpha_{2} I_{m}
$$

where $I_{m} \in \mathbb{R}^{m \times m}$ is the identity matrix.

Lemma 1 (Barbalat's Lemma [12]). Let $\phi: \mathbb{R} \rightarrow \mathbb{R}$ be a uniformly continuous function on $[0, \infty)$. Suppose that $\lim _{t \rightarrow \infty} \int_{0}^{t} \phi(\tau) \mathrm{d} \tau$ exists and is finite. Then, $\phi(t) \rightarrow 0$ as $t \rightarrow \infty$.

We assume the following:

Assumption 1. There exist three positive constants $\bar{c}_{1}, \bar{c}_{2}$, and $\bar{c}_{3}$ such that $\forall t:|\omega(t)| \leq \bar{c}_{1},|\dot{\omega}(t)| \leq \bar{c}_{2}$, and $|x(t)| \leq \bar{c}_{3}$.

We can now state the main result for the adaptive identifier.

Theorem 1 (Sensor Bias Observer). Consider the system (5) with time-varying $\omega(t)$ and $x(t)$. Let $(\hat{x}, \hat{b})$ denote the solution to (11) with $k_{1}, k_{2}>0$ positive gains, and $\omega(t)$ satisfying the Assumption 1, and is PE as defined in Definition 1. Then the equilibrium $(\Delta x, \Delta b)=(0,0)$ of (11) is globally asymptotically stable. 
Proof: From (11) and the estimation errors definition (12), the error system is

$$
\begin{aligned}
\Delta \dot{x}(t) & =-\omega(t) \times(\Delta x(t)-\Delta b(t))-k_{1} \Delta x \\
\Delta \dot{b}(t) & =-k_{2}(\omega(t) \times \Delta x) .
\end{aligned}
$$

Consider the Lyapunov candidate function

$$
\mathcal{L}=\frac{1}{2}\|\Delta x\|^{2}+\frac{1}{2 k_{2}}\|\Delta b\|^{2}
$$

where $\mathcal{L}$ is a smooth, positive definite, and radially unbounded function by construction. Taking the time derivative and recalling (14) yields

$$
\begin{aligned}
\frac{d}{d t} \mathcal{L} & =\Delta x^{\top}\left[-\omega \times(\Delta x-\Delta b)+k_{1} \Delta x\right]+\Delta b^{\top}(\omega \times \Delta x) \\
& =-k_{1}|\Delta x|^{2} \leq 0 .
\end{aligned}
$$

The time derivative of this Lyapunov function is negative semi-definite, thus guaranteeing global stability of the system, but additional arguments are needed to show global asymptotic stability. Given that the Lyapunov function (15) is bounded below by 0 and, in consequence of (16) is bounded above by its initial value, $\mathcal{L}_{t_{0}}$, and since (15) is a radially unbounded function of $\Delta x(t)$ and $\Delta b(t)$, we can conclude that $\Delta x(t)$ and $\Delta b(t)$ are bounded. Note that $\omega(t)$ is bounded from Assumption 1. From (14) and the fact that all signals on the right hand side of (14) are bounded then $\Delta x(t)$ and $\Delta b(t)$ are continuous and, in addition, $\Delta \dot{x}(t)$ and $\Delta \dot{b}(t)$ are bounded, thus $\Delta x(t)$ and $\Delta b(t)$ are uniformly continuous. For any $t \geq 0$, we have $\left(\int_{0}^{t}|\Delta x(\tau)|^{2} \mathrm{~d} \tau\right)^{1 / 2} \leq\left(\frac{1}{k_{1}} \mathcal{L}_{t_{0}}\right)^{1 / 2}$ then $\Delta x(t) \in L^{2}$. Thus from Barbalat's lemma, $\Delta x(t)$ is globally asymptotically stable at the origin, i.e. $\lim _{t \rightarrow \infty} \Delta x(t)=0$. Given that $\lim _{t \rightarrow \infty} \Delta x(t)=0$, from (14) and the boundedness of $\omega(t)$, it proves that $\lim _{t \rightarrow \infty} \Delta \dot{b}(t)=0$. Moreover, since by assumption $\omega(t)$ is PE and satisfies Assumption 1, using lemma A.1 from [4], we can conclude globally asymptotically stability for $\Delta b(t)$, i.e. $\lim _{t \rightarrow \infty} \Delta b(t)=0$.

\section{Performance Evaluation}

This section reports implementation details, discusses the evaluation methodology used to compare all the methods, and reports a comparative numerical simulation and comparative experimental evaluation of the performance of the different sensor bias estimation methods.

\section{A. Evaluation Methodology}

We compared the performance of the following six methods for the problem of sensor bias estimation. Three batch estimation methods were evaluated:

a. Centering: For comparison purposes, the sensor bias is estimated using the first step of the TWOSTEP method [2] that leads to a simple batch linear least squares solution.

b. TWOSTEP: For comparison purposes, the sensor bias is estimated using the full TWOSTEP method [2].

c. $\boldsymbol{S A R}-\boldsymbol{L S}$ : The sensor bias is estimated using the batch method proposed in section III-B based on angular-rate in the instrument-frame. The value of $\dot{x}$ is numerically calculated by a first-order numerically differentiation of $x$. Furthermore the measurements are low-pass filtered to reduce noise resulting from differentiation.

In addition, three real time methods were evaluated:

d. AI-EKF: For comparison purposes, the sensor bias is estimated using the real-time attitude independent method based on the EKF [7]. Note that for an accurate comparison the implemented version of this method only estimates the sensor bias, and not the scale and orthogonality matrix.

e. SAR-KF : The sensor bias is estimated using the Kalman filter method described in section III-C based on angularrate in the instrument-frame.

f. SAR-AID: The sensor bias is estimated using the adaptive identification method proposed in section III-D based on angular-rate in the instrument-frame.

For comparison purposes for the case of the real-time estimation methods (AI-EKF, SAR-KF and SAR-AID) the sensor measurement bias used for comparing with the batch methods consists of the average of the last $20 \%$ of the estimated sensor measurement bias.

The TWOSTEP and AI-EKF methods require knowledge of the local magnetic field magnitude. In our evaluation we used the standard US/UK World Magnetic Model for 2010-2015, [16], available online [1].

\section{B. Simulation Results}

A Monte Carlo simulation evaluation was implemented with 100 iterations for each of two datasets. The first dataset, SIM1, simulates the case of large angular movements of the instrument in all degrees of freedom, depicted in Figure 1(a). The second dataset, SIM2, simulates a constrained angular movement of the instrument, depicted in Figure 1(b). The duration of each experiment is $60 \mathrm{~s}$ and the simulated sensor data is generated at $100 \mathrm{~Hz}$. Gaussian noise was added to the measurements of the magnetometers $\left(\sigma_{m a g}=1 \mathrm{mG}\right)$ and angular-rate gyroscopes $\left(\sigma_{\text {gyro }}=5 \mathrm{mrad} / \mathrm{s}\right)$. The true magnetic field vector is $x_{0}=[200,-40,480]^{\top} \mathrm{mG}$ and the bias is $b=[20,120,90]^{\top} \mathrm{mG}$. The magnitude of the magnetic field used for the TWOSTEP and AI-EKF methods was $1 \%$ greater than the value used in generating the simulated data. We have empirically selected the gains to obtain the best performance for each method under these The covariance matrices used by the AI-EKF and SAR-KF methods are $Q=0.1 I_{6 \times 6} \mathrm{mG}$ and $R=I_{3 \times 3} \mathrm{mG}$. These values were chosen in the range of the expected process and sensor noises. The gains used by the SAR-AID method are $k_{1}=1, k_{2}=1$ for the SIM1 and $k_{1}=1, k_{2}=100$ for SIM2. These estimator gains were chosen empirically for the range of the instrument angular-rate for each experiment. Figure 2 shows the estimation performance for each simulated experiment.

The simulation results show that for a complete range of movements, SIM1, the batch methods Centered and TWOSTEP methods show the best performance. Although 


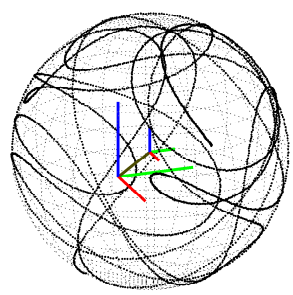

(a) Simulation Data SIM1

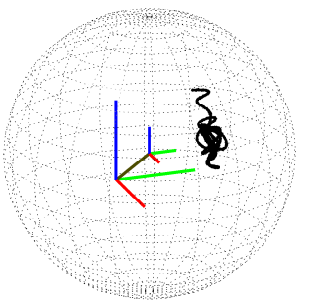

(b) Simulation Data SIM2
Fig. 1. Magnetometers simulated data: Three-dimensional plot of the magnetometers simulated data (black dots). As a reference a sphere is plotted centered at $b$. The large reference-frame is at $(0,0,0)$ with $\mathrm{x}$-axis in red, y-axis in green, and $\mathrm{z}$-axis in blue. A small reference-frame is plot at the center of the sphere.

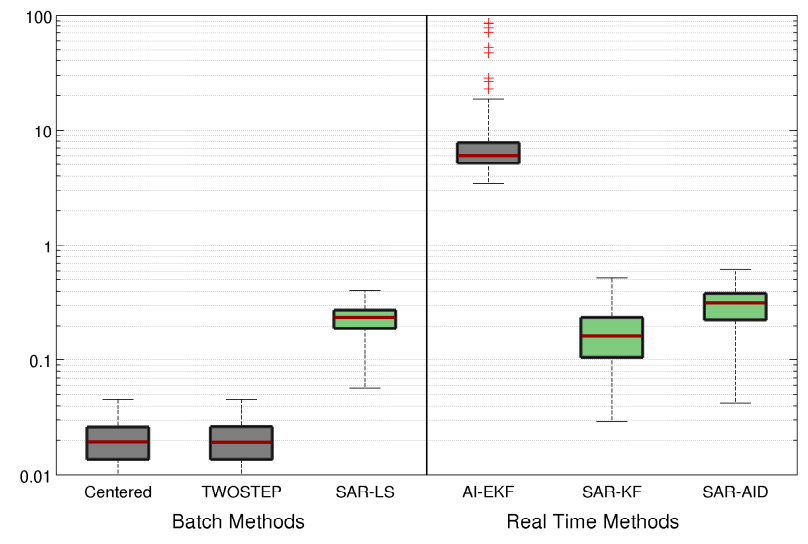

(a) Simulation results from SIM1

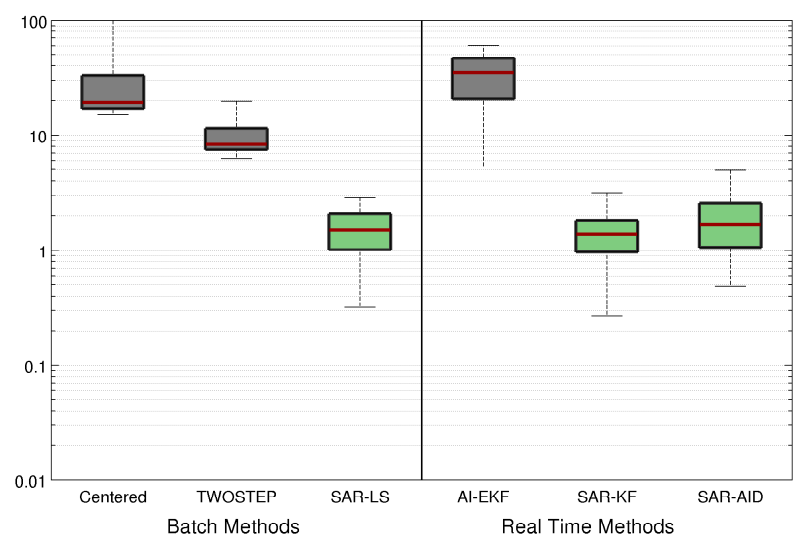

(b) Simulation results from SIM2

Fig. 2. Performance evaluation results from simulated data: The y-axis shows the sensor bias estimation error for each solution $(\mathrm{mG})$ in logarithmic scale. Each box plot is calculated from 100 iterations.

the proposed methods also estimate the magnetometer bias with an error under $1 \mathrm{mG}$. The worse performance is shown by AI-EKF method with bias estimation errors over $5 \mathrm{mG}$. The AI-EKF method is affected by linearization errors and does not ensure convergence. For the second dataset with a limited range of movements, SIM2, the SAR-LS, SAR-KF

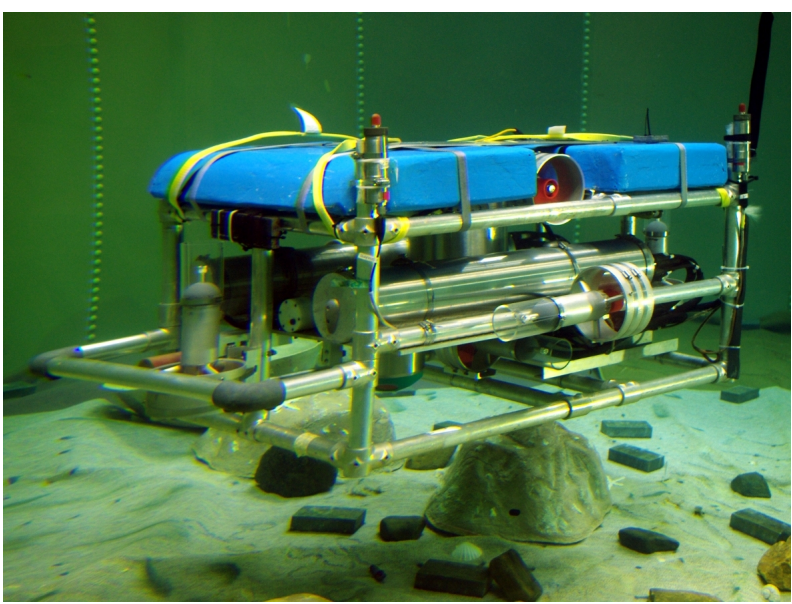

Fig. 3. JHU ROV inside the Johns Hopkins Hydrodynamic Test Facility

and SAR-AID methods show the best performance, with bias estimation errors under 2-3 mG. The other methods (Centered, TWOSTEP and AI-EKF) show a bias estimation error over 10 $\mathrm{mG}$. Note that the TWOSTEP and AI-EKF methods are very sensitive to errors in a priori, known value of the magnitude of the local magnetic field. For small movements experiments (such as SIM2), we notice that the sensor bias estimation error grows proportionally to the error in the magnitude of the magnetic field. In our simulation performance evaluation, an error in the magnitude used for estimation is introduced representing a $1 \%$ of the real magnitude. But for common applications, the difference between the local magnitude of the magnetic field and the predicted by the model can include order of magnitude higher errors (such as inside/near buildings or other unknown magnetic anomalies).

It is interesting to notice that the required processing time for SAR-KF estimation was on average 5 times greater than the SAR-AID, and 6 times greater than the SAR-LS. This is due to the fact that the Kalman filter implementation is more demanding computationally than the implementation of the adaptive identification method.

These simulation results support the utility of all proposed methods. In the next section we evaluate the performance for experimental data.

\section{Experimental Results}

This section reports the results of a comparative experimental performance evaluation of the six calibration methods in full-scale experimental trials of an remotely operated underwater vehicle (ROV) equipped with a MEMS IMU. The ROV is shown in Figure 3. The facility contains a $7.5 \mathrm{~m}$ diameter $\times 4 \mathrm{~m}$ deep indoor fresh water tank made of steel. The ROV is actuated by six $1.5 \mathrm{~kW}$ DC brushless electric thrusters and can be actively controlled in six degrees of freedom (DOF). A suite of sensors commonly employed in deep submergence underwater vehicles is present on the ROV. For our experimental performance evaluation we use a MEMS based IMU, the Microstrain 3DM-GX3-25 [17]. The internal 


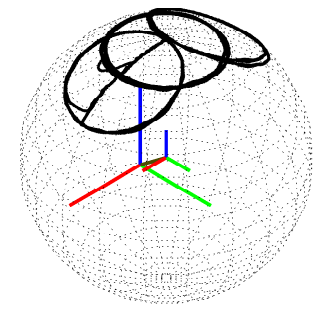

(a) Experimental Data from EXP1

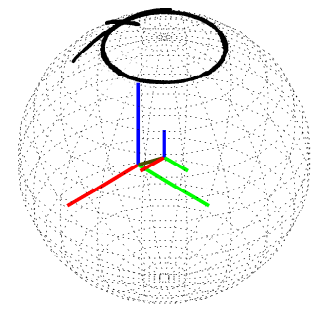

(b) Experimental Data from EXP2
Fig. 4. Experimental evaluation data: Three-dimensional plot of the magnetometers recorded data for each experiment (black dots). As a reference a sphere is plotted centered at $b^{*}$. The large reference-frame is at $(0,0,0)$ with $\mathrm{x}$-axis in red, $\mathrm{y}$-axis in green, and $\mathrm{z}$-axis in blue. A small reference-frame is plot at the center of the sphere.

magnetometer's noise level is $\sigma_{m a g}=1 \mathrm{mG}$ and angularrate gyroscope's noise level is $\sigma_{\text {gyro }}=5 \mathrm{mrad} / \mathrm{s}$. Data was sampled and recorded at $100 \mathrm{~Hz}$. For comparing the heading estimation performance, we used a high-end inertial navigation system (INS), the iXBlue PHINS III [10], as "ground-truth". The PHINS provides heading with $0.1^{\circ}$ dynamic accuracy, and pitch/roll with $0.1^{\circ}$ dynamic accuracy. The high-end INS attitude data was re-sampled to the MEMS IMU sampling time to estimate the vehicle heading position.

Two experiments were performed. The first experiment, EXP1, measure a large range of movements, Figure 4(a). The trajectory is a sequence of $720^{\circ}$ heading rotations, with different levels of pitch $\left( \pm 25^{\circ}\right)$. These trajectories are not feasible to implement in many ground, marine, or aerial vehicles. The second experiment, EXP2, measure a more feasible sequence of movements, Figure 4(a), where the range of movement of the vehicle is limited in pitch $\left( \pm 10^{\circ}\right)$ and roll $\left( \pm 5^{\circ}\right)$. In these experiments the vehicle followed pre-programmed trajectories under closed-loop control.

Although the magnetometer sensor bias is unknown for the magnetometers in this vehicle configuration, using a least square method it is possible to estimate the bias and the orthogonality scale factors based on the known attitude from the high-end INS. The analysis show that the magnetometer measurements are in an ellipsoid very close to the sphere. The calculated orthogonality matrix, $M^{*}$, and bias, $b^{*}$, for this vehicle configuration are the following

$$
M^{*}=\left[\begin{array}{ccc}
0.991 & -0.002 & -0.005 \\
0 & 0.996 & 0.018 \\
0 & 0 & 1.014
\end{array}\right] \quad b^{*}=\left[\begin{array}{c}
20.0 \\
130.5 \\
102.1
\end{array}\right] m G .
$$

For our analysis the estimation is performed for all the evaluated methods using raw measurements from the sensor including the measured orthogonality and scale factor distortions.

The magnetometers bias was estimated with each evaluated method using data from EXP1 and EXP2. From each estimated magnetometers bias, $\hat{b}_{i}$, we calculate the error with respect to $b^{*}$, although this is not a very accurate metric, due to error in $b^{*}$. As a more accurate performance comparison, we can calculate the heading error, between the heading from the magnetometers with the estimated bias $\hat{b}_{i}$ removed, $h g d_{i}$, and the heading from the high-end INS sensor, $h d g_{R E F}$. For a more accurate heading comparison, we use the same data, EXP1, although the sensor bias estimation is estimated from both datasets, EXP1 and EXP2. Table I summarizes the experimental results.
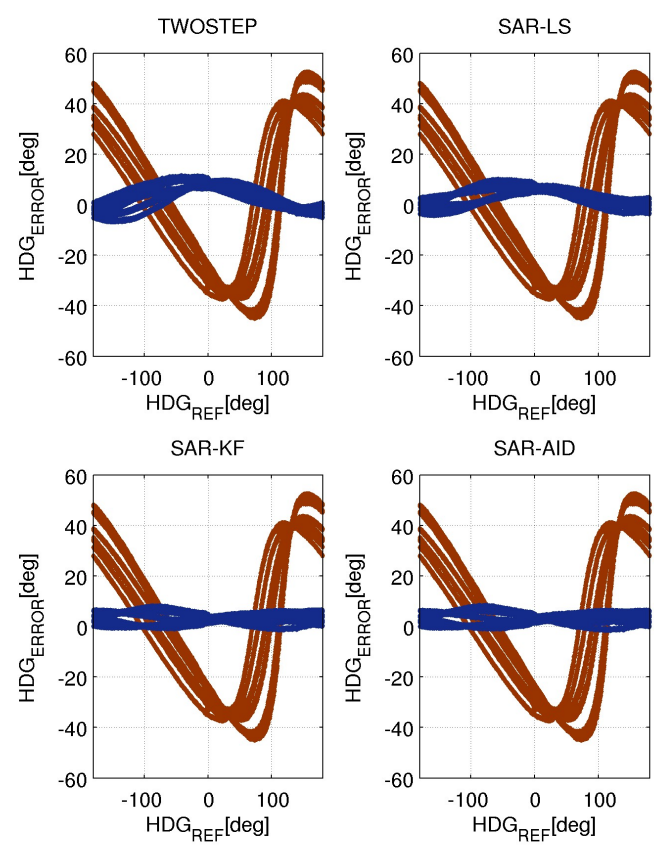

Fig. 5. Heading performance using EXP2 for estimation and EXP1 for evaluation: The $y$-axis shows the heading error for the corresponding reference heading from the high-end INS (x-axis). In light red the original heading error and in dark blue the heading error after calibration for each evaluated sensor bias estimation method. All units in degrees.

From the performance evaluation in Table I, the SARLS, SAR-KF, and SAR-AID methods show a very good performance in both experiments, with a the lowest error in the sensor bias estimation $(\sim 10 \mathrm{mG})$, and excellent performance correcting the heading error from the original $27^{\circ}$ range to less than $2^{\circ}$ in some cases. On the other hand, the Centered and TWOSTEP methods while show a good performance for the first large angular movements experiment, these methods have a less accurate performance for a more limited calibration dataset. For EXP2, the Centered and TWOSTEP methods show error that is half of the original error with EXP1. AI-EKF, like in the numerical simulations, shows the worse performance of the evaluated methods, although is still able to estimate the bias and improve the heading estimation, but not as good as the proposed methods (SAR-LS, SAR-KF, and SAR-AID). Figure 5 shows the heading error for the TWOSTEP method and the three proposed methods (SAR- 
TABLE I

SUMMARY OF THE SENSOR BIAS ESTIMATION RESULTS FROM EXP1 AND EXP2. BEST TWO PERFORMANCES IN EACH RELEVANT COLUMN IS MARKED IN BOLD FONT.

\begin{tabular}{l|ccc|c|c|ccc|c|c}
\hline & \multicolumn{1}{|c}{ EXP1 } & \multicolumn{6}{c}{ EXP2 } \\
\hline & $\begin{array}{c}\hat{b}_{x} \\
{[\mathrm{mG}]}\end{array}$ & $\begin{array}{c}\hat{b}_{y} \\
{[\mathrm{mG}]}\end{array}$ & $\begin{array}{c}\hat{b}_{z} \\
{[\mathrm{mG}]}\end{array}$ & $\begin{array}{c}\left|\hat{b}-b^{*}\right| \\
{[\mathrm{mG}]}\end{array}$ & $\begin{array}{c}\sigma\left(h d g_{E}\right) \\
{[\mathrm{degrees}]}\end{array}$ & $\begin{array}{c}\hat{b}_{x} \\
{[\mathrm{mG}]}\end{array}$ & $\begin{array}{c}\hat{b}_{y} \\
{[\mathrm{mG}]}\end{array}$ & $\begin{array}{c}\hat{b}_{z} \\
{[\mathrm{mG}]}\end{array}$ & $\begin{array}{c}\left|\hat{b}-b^{*}\right| \\
{[\mathrm{mG}]}\end{array}$ & $\begin{array}{c}\sigma\left(h d g_{E}\right) \\
{[\mathrm{degrees}]}\end{array}$ \\
\hline Raw & 0.000 & 0.000 & 0.000 & 166.897 & 27.362 & 0.000 & 0.000 & 0.000 & 166.897 & 27.362 \\
\hline Centered [2] & 21.945 & 123.870 & 85.770 & 17.732 & $\mathbf{2 . 3 7 7}$ & 18.242 & 136.132 & 79.734 & 23.132 & 4.719 \\
\hline TWOSTEP [2] & 21.940 & 123.867 & 85.668 & 17.826 & $\mathbf{2 . 3 7 8}$ & 18.246 & 136.184 & 78.541 & 24.298 & 4.753 \\
\hline SAR-LS & 21.050 & 130.370 & 94.302 & $\mathbf{7 . 8 7 0}$ & 3.340 & 19.895 & 131.036 & 98.571 & $\mathbf{3 . 5 7 1}$ & 3.435 \\
\hline AI-EKF [7] & 17.490 & 122.283 & 9.244 & 93.253 & 5.547 & 18.762 & 138.095 & 12.162 & 90.266 & 7.409 \\
\hline SAR-KF & 21.486 & 130.041 & 100.846 & $\mathbf{1 . 9 9 8}$ & 3.238 & 21.501 & 118.953 & 104.425 & 11.874 & $\mathbf{1 . 8 9 7}$ \\
\hline SAR-AID & 21.265 & 130.364 & 93.475 & 8.718 & 3.349 & 21.539 & 119.456 & 105.742 & $\mathbf{1 1 . 7 3 0}$ & $\mathbf{1 . 9 2 3}$ \\
\hline
\end{tabular}

LS, SAR-KF, and SAR-AID).
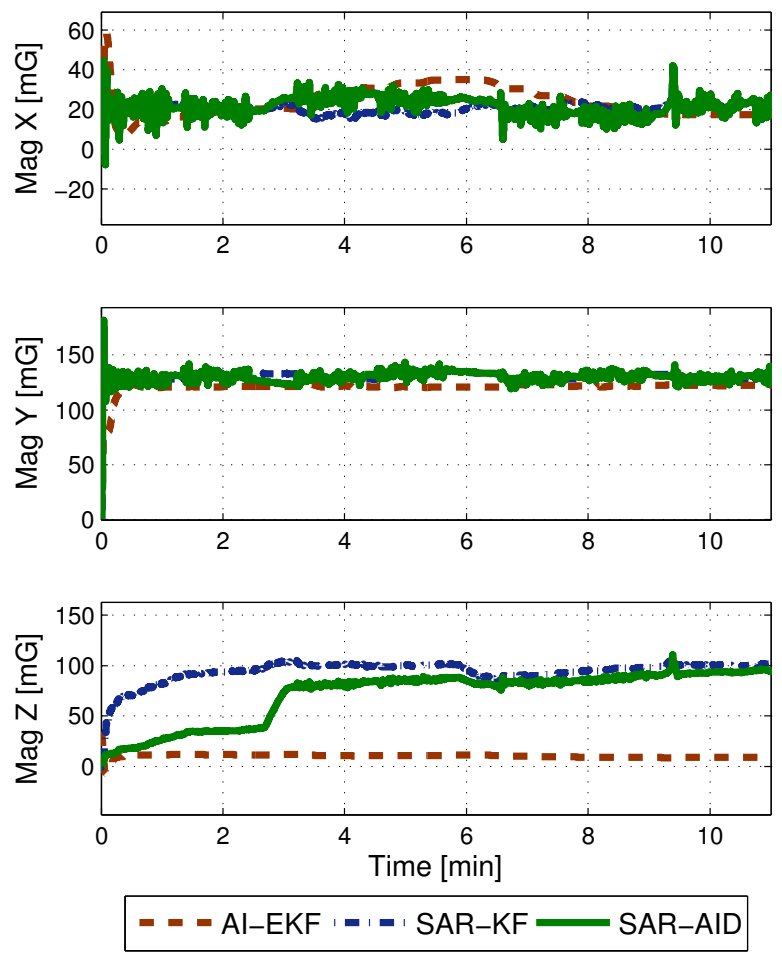

Fig. 6. Magnetometer bias estimation over time for EXP2: The x-axis shows the time (min) and the $y$-axis the estimated bias for each method (mG). In segmented red the AI-EKF method, in segmented dot blue the SAR-KF method, and in solid green the SAR-AID method.

Figure 6 shows the sensor bias estimation over time for the three real time evaluated methods (AI-EKF, SAR-KF, and SAR-AID) for EXP2. The results show that all the methods converge quickly in $b_{x}$ and $b_{y}$ due to the nature of the excitation with large heading movements. But the term $b_{z}$, is correctly estimated only for SAR-EKF and SAR-AID but not for AI-EKF.

\section{CONCLUSIONS}

The proposed angular-rate aided estimation methods (SARLS, SAR-KF, and SAR-AID) were shown to improve the sensor bias estimation performance under some circumstances when compared with the previously reported methods (Centered, TWOSETP, and AI-EKF).

The numerical simulation and experimental results quantified the sensor measurement bias estimation performance under different scenarios of calibration motions. The proposed method, SAR-LS, SAR-KF and SAR-AID, show good performance for all the evaluated scenarios. The previously reported methods, Centered, TWOSETP, and AI-EKF, show good performance only when the data represent large instrument angular motion. In addition, TWOSTEP and AI-KF methods require exact knowledge of the magnitude of the local magnetic field vector, and are very sensitive to error in this value.

The comparative experimental evaluation quantified the resulting calibrated heading estimation performance when compared with the heading reported by a high-end INS. The proposed method, SAR-LS, SAR-KF and SAR-AID, show significantly smaller heading error after calibration than all the previously reported methods for the case of a more feasible sequence of calibration movements for most ground, marine, or aerial vehicles. Finally the proposed methods (SAR-LS, SAR-KF, and SAR-AID) ensure convergence to the estimated sensor bias value while the real time method, AI-EKF, does not ensure convergence to the true values.

In the future, we plan to improve the gain selection for some of the proposed methods and to perform further analysis under different conditions and applications.

These results may be useful to improve the performance of low-cost MEMS-based attitude sensors and, in consequence, improve the navigation accuracy for low-cost ground, marine, or aerial vehicles.

\section{ACKNOWLEDGMENT}

Our sincere thanks to our reviewers. This work was supported by the National Science Foundation under NSF award IIS-0812138. Support for the first author was also provided by a Fulbright/Conicyt Fellowship. 


\section{REFERENCES}

[1] The World Magnetic Model. National Oceanic and Atmospheric Administration (NOAA). http://www.ngdc.noaa.gov/geomag/WMM/ DoDWMM.shtml.

[2] R. Alonso and M. D. Shuster. TWOSTEP, a fast robust algorithm for attitude-independent magnetometer bias determination. The Journal of the Astronautical Sciences, 50(4):433-451, Dec. 2002.

[3] R. Alonso and M. D. Shuster. Complete linear attitude-independent magnetometer calibration. Journal of the Astronautical Sciences, 50(4):477490, 2002

[4] G. Besancon. Remarks on nonlinear adaptive observer design. Systems and Control Letters, 41(4):271 - 280, 2000.

[5] N. Bowditch. American practical navigator. Number v. 1 in H.O. pub. Govt. print. off., 1984.

[6] J. Crassidis, F. Markley, and Y. Cheng. Survey of nonlinear attitude estimation methods. Journal of Guidance Control and Dynamics, 30(1):12, 2007.

[7] J. L. Crassidis, K. L. Lai, and R. R. Harman. Real-time attitudeindependent three-axis magnetometer calibration. Journal of Guidance Control and Dynamics, 28(1):115-120, 2005.

[8] B. Gambhir. Determination of magnetometer biases using module RESIDG. Computer Sciences Corporation, Report No.3000-3270001TN. Technical report, march 1975 .

[9] P. Guo, H. Qiu, Y. Yang, and Z. Ren. The soft iron and hard iron calibration method using extended Kalman filter for attitude and heading reference system. In Position, Location and Navigation Symposium, 2008 IEEE/ION

[10] IXSEA. PHINS III User Guide. IXSEA, MU-PHINSIII-002 A edition, July 2008
[11] R. Kalman. A new approach to linear filtering and prediction problems. Journal of Basic Engineering, 82(1):35-45, 1960.

[12] H. Khalil. Nonlinear Systems. Prentice Hall, 2002.

[13] M. Kok, J. D. Hol, T. B. Schon, F. Gustafsson, and H. Luinge. Calibration of a magnetometer in combination with inertial sensors. In Information Fusion (FUSION), 2012 15th International Conference on, pages 787-793, July 2012.

[14] X. Li and Z. Li. A new calibration method for tri-axial field sensors in strap-down navigation systems. Measurement Science and Technology, 23(10):105105, Oct. 2012.

[15] G.-F. Ma and X.-Y. Jiang. Unscented Kalman filter for spacecraft attitude estimation and calibration using magnetometer measurements. In Machine Learning and Cybernetics, 2005. Proceedings of 2005 International Conference on, volume 1, pages 506-511, 2005.

[16] S. Maus, S. Macmillan, S. McLean, B. Hamilton, A. Thomson, M. Nair, and C. Rollins. The US/UK World Magnetic Model for 2010-2015. Technical report, December 2010.

[17] Microstrain Inc. 3DM-GX3-25 miniature attitude heading reference system datasheet. Technical Report P/N 8400-0033 rev. 002, Microstrain Inc., Williston, VT, 2012.

[18] S. Sastry and M. Bodson. Adaptive Control: Stability, Convergence, and Robustness. Prentice Hall Information and System Sciences Series. Prentice Hall, 1989.

[19] S. Thrun, W. Burgard, and D. Fox. Probabilistic Robotics (Intelligent Robotics and Autonomous Agents). The MIT Press, 2005.

[20] J. F. Vasconcelos, G. Elkaim, C. Silvestre, P. Oliveira, and B. Cardeira. Geometric approach to strapdown magnetometer calibration in sensor frame. Aerospace and Electronic Systems, IEEE Transactions on, 47(2):1293-1306, Apr. 2011. 